\title{
A criação do MIBRELA, um software brasileiro de orientação para pacientes com esclerose lateral amiotrófica
}

\author{
MIBRELA, orientation Brazilian software for amyotrophic lateral sclerosis \\ patients.
}

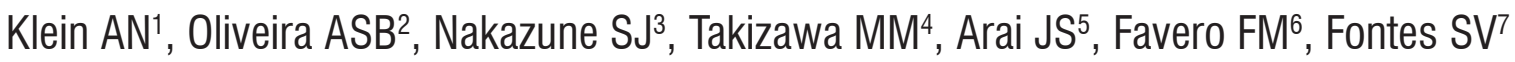

\section{RESUMO}

Objetivo: Elaborar uma nova estratégia por meio de um programa de computador (software) para ministrar orientações preventivas e facilitadoras para o processo de reabilitação de pacientes com ELA. Método: A elaboração do produto MIBRELA (manual interativo brasileiro de esclerose lateral amiotrófica), ou seja, um software brasileiro de orientações, para pacientes com esclerose lateral amiotrófica foi realizada a partir da aplicação de um manual impresso, previamente elaborado pela própria autora deste estudo, e testado em 114 pacientes com diagnóstico provável ou definitivo de esclerose lateral amiotrófica que foram assistidos no serviço de terapia ocupacional do Setor de Doenças Neuromusculares da UNIFESP. Resultados: este manual consiste em um produto do tipo CD (compact disk) e, trata-se de um aplicativo Desktop baseado em telas (janelas), sendo necessário somente um computador simples sem acesso a rede ou internet, mas com os pré-requisitos instalados: .NET Framework 1.1 e sistema operacional Windows 98/ME/2000/XP No total, o usuário do programa tem 102 opções de interatividade, incluindo fotos, definições, explicações de determinados produtos e equipamentos, orientações de cuidados e precauções durante a realização de alguma atividade entre outros. Conclusão: Este software contem as informações necessárias para facilitar as orientações preventivas e reabilitadoras de pacientes com ELA.

\section{Unitermos: Software, Esclerose amiotrófica lateral, Informática médica.}

Citação: Klein AN, Oliveira ASB, Nakazune SJ, Takizawa MM, Arai JS, Favero FM, Fontes SV. A criação do MIBRELA, um Software brasileiro de orientação para pacientes com esclerose lateral amiotrófica. Rev Neurocienc 2007; 15(1):44-48.

\section{SUMMARY}

Objective: The aim of the study was to elaborate a new strategy from a program of computer (Brazilian Software) to facilitate orientation for the process of rehabilitation of amyotrophic lateral sclerosis (ALS) patients. Method: The elaboration of a product Mibrela (Brazilian Manual Interactive from ALS), a Brazilian software for orientations to ALS patients, was realized based on a printed manual elaborated previous by the authors from this study and applied on 114 patients with probably or definitive ALS diagnostic that was assisted in the Occupational Therapy Service of the Sector of Neuromusculares Disease at UNIFESP. Results: This manual consists in a CD (compact disk) that is applicable Desktop based on screens. It is necessary just one simple computer without Internet access, but with some programs inside .NET Framework 1.1 and operation system from Windows 98/ME/2000/XP. Finaly the program user has 102 options of announces, including pictures, definitions, explanations of some products

Trabalho realizado no Setor de Doenças Neuromusculares da UNIFESP.

1. Terapeuta Ocupacional, especializada em doenças reumáticas e neuromusculares pela UNIFESP, responsável pelo Setor de Terapia Ocupacional do Ambulatório de esclerose lateral amiotrófica da UNIFESP.

2. Professor afiliado da Disciplina de Neurologia da UNIFESP

3. Terapeuta ocupacional especializanda em Terapia Ocupacional em Reabilitação pela UNIFESP.

4. Bacharel em Ciências da Computação.

5. Bacharel em Ciências da Computação.

6. Fisioterapeuta, Mestra em Ciências pela UNIFESP

7. Fisioterapeuta da Disciplina de Neurologia da UNIFESP e Doutora em Ciências pela UNIFESP. 
and equipment, orientations and aids during some activities. Conclusion: This software is has the necessary information to facilitate the orientations about prevention and rehabilitation for ALS patients.

\section{Keywords: Software, Amyotrophic lateral sclerosis, Medical informatics.}

Citation: Klein AN, Oliveira ASB, Nakazune SJ, Takizawa MM, Arai JS, Favero FM, Fontes SV. MIBRELA, orientation Brazilian software for amyotrophic lateral sclerosis patients. Rev Neurocienc 2007; 15(1):44-48.

\section{INTRODUÇÃO}

Esclerose lateral amiotrófica (ELA) é uma doença neurodegenerativa caracterizada por perda progressiva dos neurônios motores do córtex, do tronco cerebral e do corno anterior da medula espinal, levando à atrofia e a fraqueza muscular generalizada ${ }^{1}$.

A incidência anual é de 2:100.000 pessoas e a prevalência estimada é de 6 por 100.000 habitantes, atinge mais comumente homens na proporção de 3:2 e com pico de incidência na quinta década de vida² .

O paciente com ELA é confrontado com uma doença de origem desconhecida, cura improvável e prognóstico incerto ${ }^{3}$. Há, freqüentemente frustração com a rapidez da perda muscular e o aumento da incapacidade ${ }^{4}$, havendo necessidade emergente de orientações eficientes por equipe multiprofissional.

Existem muitos cuidados a serem incluídos no cotidiano desses pacientes como, por exemplo, fazer uso dos medicamentos prescritos, modificar hábitos em relação às atividades de vida diária (AVD), ajustar-se às novas circunstâncias socioeconômicas, enfrentar conseqüências emocionais e participar de decisões referentes ao tratamento. Em contrapartida, é responsabilidade do sistema de saúde preparar os pacientes para estes cuidados, sendo uma das estratégias utilizadas o investimento na educação dos mesmos ${ }^{5}$.

A educação em saúde, perante um processo nosológico pode resultar em benefícios sob vários aspectos, como na melhoria dos sintomas clínicos, na manutenção e adaptação às limitações físicas e funcionais decorrentes deste e, também no aspecto emocional, facilitando a aceitação da doença, podendo interferir favoravelmente no estilo de vida. Além disso, os programas de educação do paciente promovem diminuição dos custos diretos e indiretos do sistema de saúde, por meio da redução da necessidade do número de visitas ao médico e do uso de hospitais 5 .

No entanto, o método educacional do paciente e cuidador mais eficiente ainda não está claro ${ }^{5,6}$. Essas orientações educacionais são ministradas, geralmente, por meio de comunicação verbal que, muitas vezes não são totalmente compreendidas e ou assimiladas. A informação escrita representa um importante adjuvante neste processo, desde que seja compreendida de maneira clara pela população-alvo ${ }^{7}$. O estudo de outras estratégias educacionais que poderiam propiciar um número maior de acesso às informações, nos diferentes estágios da doença faz-se necessário, para investigar qual método poderia facilitar maior aderência às orientações.

Nos últimos anos, o acesso cada vez mais fácil à internet tem levado os pacientes e familiares a procurar e obter informações de saúde por esse meio, podendo este ser uma alternativa interativa ${ }^{8}$.

A abordagem de uma doença complexa, como a ELA requer comunicação efetiva para a transmissão de informação e cuidado de saúde 4 . Os pacientes são tratados, geralmente em ambiente ambulatorial e o tempo de atendimento pode não ser o suficiente para que as informações sobre a doença e seu manejo sejam transmitidas por toda equipe multidisciplinar envolvida, e de maneira eficiente ${ }^{5}$.

Sendo assim, o objetivo deste estudo é elaborar uma nova estratégia por meio de um programa de computador (software) para ministrar orientações preventivas e facilitadoras para o processo de reabilitação de pacientes com ELA.

\section{MÉTODO}

A elaboração do produto MIBRELA (manual interativo brasileiro de esclerose lateral amiotrófica), ou seja, um software brasileiro de orientações, para pacientes com esclerose lateral amiotrófica foi realizada a partir da aplicação de um manual impresso, previamente elaborado pela própria autora deste estudo, e testado em 114 pacientes com diagnóstico provável ou definitivo de esclerose lateral amiotrófica que foram assistidos no serviço de terapia ocupacional do Setor de Doenças Neuromusculares da UNIFESP.

\section{Aplicação do manual impresso}

O manual impresso contém 47 informações descritivas de técnicas sobre conservação de energia, indicações de adaptações, orientações do uso adequado de órteses para os membros superiores (MMSS), mudanças ergonômicas no domicílio e, prescrição de sistemas de adequação postural.

Estas informações estão organizadas em sete domí- 
nios, e podem ser assinaladas ou não, pelo terapeuta de acordo com a necessidade individual de cada paciente: 1) Alimentação: engrossar o talher, entortar o cabo da colher, elevar o prato para diminuir o ângulo entre a mesa e a boca, utilizar copo leve de preferência de plástico e com alça grande, utilizar canudo, recortar uma meia lua no copo para evitar hiperextensão do pescoço, pregar dois pregos em uma tábua de madeira para facilitar o corte de alimentos, deixar todos os objetos necessários ao alcance da mão; 2) Higiene: elevar o assento do vaso sanitário, instalar barras de apoio próximo ao vaso sanitário e dentro do box do chuveiro, utilizar cadeira de banho higiênica, utilizar escova de dente elétrica, instalar bidê ou duchas específicas no vaso sanitário, engrossar escova de dente e aparelho de barbear, alongar e ou engrossar cabo de pente ou escova de cabelo, sentarse para escovar os dentes ou fazer a barba apoiando os cotovelos na borda da pia, utilizar um espelho atrás da torneira, sentar-se para tomar banho - gasto energético é menor, colocar o "espaguete de piscina" na parte posterior da cadeira de banho, para aumentar a superfície de apoio do tronco e pescoço, colocar o sabonete dentro de uma meia calça (recorte de uma perna) e amarrar no registro do chuveiro ou na cadeira de banho; 3) Vestuário: colocar "aro de chaveiros" nos zíperes, substituir botões por velcro, utilizar prolongador (madeira de 80 cm, "gancho de planta", imã, calçadeira), utilizar colocador de meia, utilizar duas cadeiras para auxiliar na colocação e retirada da roupa, iniciar a retirada e colocação da blusa pelo lado que possui maior dificuldade, utilizar calçadeira alongada, Substituir calçados com cadarço por outros modelos; 4) Comunicação: prancheta para escrita, figuras do cotidiano, adaptador para indicar figura, utilizar apoio de bíblia para apoiar o livro (almofada, posição de leitura em direção ao horizonte); 5) Lazer e informática: adaptadores de teclado e mouse (customizados), utilizar teclado virtual (programa do próprio computador), aumentar a sensibilidade do teclado (programa de computador), utilizar apoio de baralho, utilizar mesa regulável e/ou recortada em meia-lua com apoio para os cotovelos (para diversas atividades), engrossar ou adaptar caneta e ou pincel, utilizar adaptador de chave; 6) Posicionamentos: elevar a cama, sofá ou cadeira, sentar-se próximo à parede para apoiar a cabeça no travesseiro, utilizar colar cervical (olhar a indicação das horas), utilizar bolsa, bolsos ou cadeira para apoio de membros superiores, não deixando os MMSS sem apoio (técnica de proteção articular), órteses de membros superiores (utilizar duas horas na parte da manhã, duas horas na parte da tarde, para dormir a noite toda e, para atividades específicas); 7) Locomoção (descrição detalhada da locomoção).

A amostra de pacientes que este manual impresso foi testado é composta por 75 homens e 39 mulheres e, com média de idade de 56 anos e, a aplicação do mesmo foi realizada em uma sessão de terapia ocupacional
(TO) ambulatorial, com base em avaliação funcional previamente realizada por um único avaliador e, após três meses foram reavaliados, a fim de verificar se houve ou não aderência às orientações ministradas.

A verificação da aderência ocorreu com a pergunta dicotômica de sim ou não para cada um dos itens que foram orientados individualmente.

Dos 114 pacientes que foram avaliados e receberam as orientações com o manual impresso, 46\% não retornaram para a reavaliação, 54\% dos pacientes que retornaram, e seguiram as orientações do TO, a aderência foi de $93 \%$. Os principais motivos relatados pelos $4 \%$ dos pacientes que não aderiram foram: esquecimentos, não obtiveram ajuda de cuidadores ou familiares para colocar em prática alguma orientação ou não consideraram-nas necessárias.

\section{RESULTADOS}

\section{Elaboração do Software}

Utilizando como base os resultados da aplicabilidade do manual impresso foi elaborado o produto MIBRELA, ou seja, o material de orientação impressa foi transformado em um programa de computador com fotos ilustrativas e legendas explicativas, criando um manual interativo para pacientes com ELA, seus cuidadores e para profissionais da saúde que os assistem.

Para criação deste produto foi necessária a colaboração de dois bacharéis em ciência da computação que através de seus conhecimentos na área de tecnologia da informação programaram o software.

O MIBRELA é um aplicativo para sistemas operacionais Windows desenvolvido com a linguagem de programação C\# (C Sharp) sobre a plataforma Microsoft .NET. Esta é uma iniciativa da Microsoft que visa uma plataforma única para desenvolvimento e execução de sistema e aplicações, assim qualquer código desenvolvido para .NET pode ser executado em qualquer dispositivo, desde que possua um framework, o .NET Framework. $^{9}$

A linguagem C\# é uma linguagem de programação orientada a objetos, baseada nas linguagens $\mathrm{C}++\mathrm{e}$ Java, criada pela Microsoft que permite a construção de sistema e aplicações para plataforma Microsoft. NET.9

\section{O software}

Este manual consiste em um produto do tipo CD (compact disk) e, trata-se de um aplicativo Desktop baseado em telas (janelas), sendo necessário somente um computador simples sem acesso a rede ou internet, mas com todos os pré-requisitos especifícados por aqueles que os desenvolveram instalados, sendo eles: .NET Framework 1.1 e sistema operacional Windows 98/ME/2000/XP9 


\section{Utilização}

Assim que o CD (compact disk) que contém o programa é inserido no computador, há uma página inicial de instalação do mesmo, por meio do .NET Framework 1.1 para sistema operacional Windows 98//Me/2000/XP. Após esta etapa, o software pode ser aberto e, uma página-guia inicial contém as explicações de como o usuário deve interagir, havendo opção de três janelas:

Na primeira janela (Figura 1) o usuário poderá interagir com seis atividades específicas: alimentação, higiene, vestuário, comunicação, locomoção e trabaIho/lazer. Ao identificar a sua dificuldade na execução destas tarefas, poderá encontrar diversas soluções e intervenções mais comumente utilizadas para facilitar estas ações.

Ao escolher a segunda janela (Figura 2), o usuário poderá interagir com um homúnculo, e conforme o mouse é deslocado para determinadas regiões desta figura humana (pescoço, ombros, mãos e tronco) aparecerá as principais soluções relacionadas ao uso de diversos tipos de órteses e dispositivos de adequação postural.

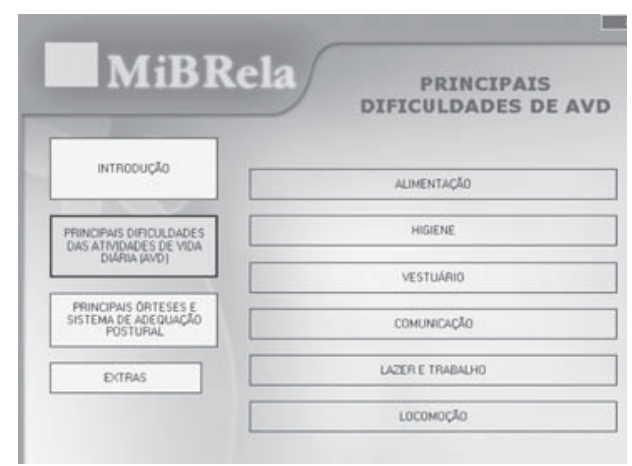

Figura 1. Nesta janela o usuário poderá interagir com seis domínios (alimentação, higiene, vestuário, comunicação, lazer/ trabalho e locomoção), identificando as principais dificuldades apresentadas nestas atividades de vida diária.

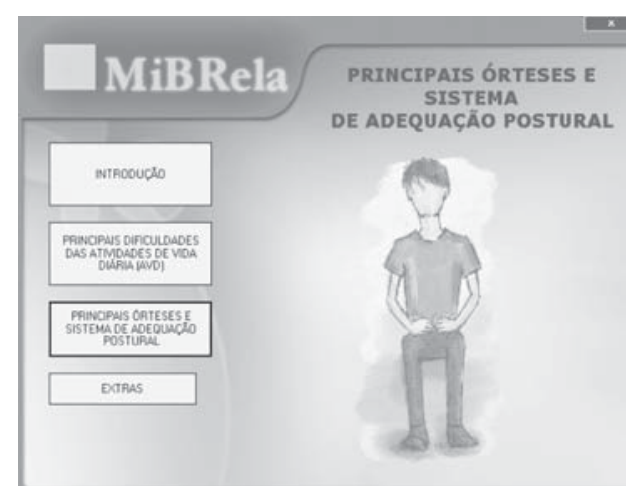

Figura 2. Nesta página o usuário poderá deslizar o mouse sobre um homúnculo, escolhendo algumas regiões comumente acometidas por pacientes com ELA (pescoço, ombros, tronco e mãos), e assim que clicar sobre a parte do corpo, aparecerá as principais órteses e dispositivos de adequação postural utilizadas
Já a proposta da terceira janela é apresentar alguns depoimentos de pacientes que passaram por avaliação e intervenção de TO e que elaboraram algum tipo de equipamento, ou técnica facilitadora e, gostariam de compartilhar este conhecimento inovador com os usuários deste programa.

No total, o usuário do programa tem 102 opções de interatividade, incluindo fotos, definições, explicações de determinados produtos e equipamentos, orientações de cuidados e precauções durante a realização de alguma atividade entre outros.

\section{DISCUSSÃO}

De acordo com a porcentagem de pacientes que não retornaram para reavaliação trimestral, e com os que não aderiram ao manual de orientações impresso, podemos inferir que o uso de um instrumento como o software poderia ser capaz de reforçar as orientações realizadas pelo TO, mesmo quando o paciente não retorna para o acompanhamento ambulatorial. Quanto aos pacientes que aderiram as orientações propostas no manual impresso, o software poderia aumentar o acesso às informações, melhorando a eficiência de sua utilização no cotidiano.

Há várias hipóteses que justificam um baixo índice de retorno às consultas ambulatoriais dos pacientes com ELA como, por exemplo, a dificuldade do sistema de transporte para o deslocamento do paciente de seu domicílio até o hospital; há necessidade de acompanhamento de terceiros devido o grau de dependência da maioria dos pacientes. Estes acompanhantes, muitas vezes, não podem faltar em seus empregos para acompanhá-los às consultas; muitos pacientes não possuem um sistema de cadeira de rodas adequado, o que torna difícil a locomoção destes e, geralmente estas consultas demandam um longo período nas salas de espera, o que pode propiciar desconforto aos mesmos.

Estudos realizados em pacientes com doenças reumatológicas, avaliando o efeito da educação de pacientes com artrite reumatóide por meio de programa de computador, mostraram diferenças estatisticamente significativas entre os grupos avaliados ${ }^{10}$. O grupo que utilizou o computador obteve mais conhecimento do que o grupo controle, e mostrou-se mais esperançoso diante do prognóstico da doença e foi capaz de modificar comportamentos, como o de utilizar técnicas de proteção articular no dia-a-dia. Em outro estudo multicêntrico, que avaliou a eficácia de um programa de computador que continha informações sobre osteoartrose e o uso de medicamento, mostrou que este programa foi capaz de diminuir significativamente o uso inapropriado do medicamento, além do aumento da aderência ${ }^{11}$.

Não foram encontrados, na literatura estudos sobre educação em saúde para pacientes com ELA utilizan- 
do-se de programas de computador. Vale ressaltar que é muito comum pacientes com ELA, devido à incapacidade física utilizarem computador como um meio facilitador de comunicação, troca de experiências, e redução do isolamento ${ }^{12}$. A tecnologia envolvida em diversos dispositivos potencializadores de função, para o uso do computador em pacientes com ELA como, por exemplo, mouses sensíveis e teclados virtuais tem sido recomendada e utilizada, o que pode facilitar o uso de produtos como o manual de informações e ou orientações de cuidados do tipo programas de computador.

Diversas associações específicas de doenças do neurônio motor, ao redor do mundo, incluindo a Associação Brasileira de Esclerose Lateral Amiotrófica (ABRELA), disponibilizam vários tipos de manuais educativos impressos para paciente, cuidadores e profissionais. Na ALS Association ${ }^{13}$ estes métodos educativos, estão disponíveis na Internet ou podem ser adquiridos comercialmente em fitas de VHS, mas foram elaborados em língua estrangeira, o que pode dificultar a utilização destes pelos pacientes com ELA brasileiros.

Devido a ELA ser uma doença, na maioria dos casos, rapidamente progressiva, há necessidade de várias informações específicas de acordo com a fase da doença e, devem ser ministradas em curtos intervalos de tempo, devendo estas informações serem eficientes e reforçadas ${ }^{3,4}$. Sendo assim, transformar o manual impresso em um programa de computador interativo talvez possa ser uma estratégia vantajosa para orientação de pacientes com ELA, devendo este ser testado em um estudo controlado para averiguar seus reais efeitos.

\section{CONSIDERAÇÕES FINAIS}

Por meio deste software brasileiro, o MIBRELA, as informações individuais, realizadas no setor de terapia ocupacional podem ser transmitidas de maneira ilustrativa, aumentando o acesso às medidas facilitadoras, preventivas e reabilitadoras. Este manual não descarta a avaliação de terapeuta ocupacional, mas facilita o conhecimento de sistemas, produtos e equipamentos customizados ou adquiridos comercialmente com o objetivo de melhorar a qualidade de vida dos pacientes brasileiros com ELA.

\section{REFERÊNCIAS BIBLIOGRÁFICAS}

1. Miller RG, Sufit R. New Approaches to the treatment of ALS. Neurol 1997;48(41):S28-S32.

2. Jenkinson $\mathrm{C}$, Swash M, Fitzpatrick R. The Europen amyotrophic lateral sclerosis health profile study. J neurol scienc 1998;160(1):S122-126.

3. BrooksBR, Shodis KA, Lewis DH, Rawling JD, Sanjak M, Belden DS, et al. Natural history of amyotrophic lateral sclerosis: Quantification of symptoms, signs, strength, and function. In:Serratrici, G.T.;Munsat, T.L.Advances in neurology. Philadephia: Raven Publishers, v68, 1995, p163-183.

4. McDonald ER, Wiedenfeld SA, Hillel A, Carpenter CL, Walter RA. Survival in amiotrophic lateral sclerosis. The role of psychological factors. Arch Neurol $1994 ; 51: 17-23$.

5. Holman H, Lorig K. Perceived self-efficacy in self-management of chronic disease. In: Scharzer R (ed). Self-efficacy: Thought control of action. Washington-DC: Hemisphere Publishing Corporation, 1992.

6. Lorish CD, Boutaugh ML. Patient education in Rheumatology. Curr Opin Rheumatol 1997; 9:106-111.

7. Hill J. A practical guide to patient education and information giving. Bailliere's
Clinical Rheumatology1997; 11:109-127.

8.Edworthy SM. Crawling through the web: What do our patients find? J Rheumatol 2001;28(1):185-191.

9. Watson K. Beginning C \#: Programando. São Paulo: Makron Books, 2002.

10. Wetstone SL, Sheehan TJ, Votanw RG, Peterson MG, Rothfield N. Evaluation of a computer based education lesson for patients with rheumatoid arthritet. J Rheumatol 1985;12:5.

11. Edworthy SM, Devins GM. Improving medication aderence through patient education distinguishing between appropriate and inappropriate utilization. J Rheumatol 1999;26:8.

12. O'Brien MR, Clark D. Personal experiences of living with ALS/MND: illness narratives posted on the "Web". Amyotrophic Lateral Scler Other Motor Neuron Disord 2005;6:160.

13. Living with ALS Manuals (Endereço na Internet). ALS Association (Atualizado em: 16/04/2007; Acessado em 24/04/2007). Disponível em:

http://www.alsa.org/resources/manuals.cfm?CFID $=3853090 \& C F T O K E N=9$ 1316142 